\title{
A data-centric model for smartphone security
}

\begin{abstract}
Recently the usage of mobile devices has increased rapidly and it will be growing even more with enhancements in mobile device's functionality. Due to their enhanced functionality, mobile devices are used in corporate businesses and carry valuable information such as corporate transactions. So the security of mobile devices needs to be improved.This paper discusses various vulnerabilities of different components and functionalities of mobile devices such as SMS, wireless and applications. Moreover, mobile malware threats are explained and their effects are studied. Finally, a data-centric model to protect all the vulnerabilities and prevent application and malware threats is suggested. The model is able to ensure confidentiality, integrity and availability of data stored on mobile devices.
\end{abstract}

Keyword: Mobile devices; Data-centric; Data loss prevention; Malware threat; Smartphone 